\title{
Analysis of integrodifference equations with a separable dispersal kernel
}

\author{
Jason Bramburger • Frithjof Lutscher
}

Received: date / Accepted: date

\begin{abstract}
Integrodifference equations are a class of infinite-dimensional dynamical systems in discrete-time that have recently received great attention as mathematical models of population dynamics in spatial ecology. The dispersal of individuals between generations is described by a 'dispersal kernel', a probability density function for the distance that an individual moves within a season. Previous authors recognized that the dynamics are reduced to a finitedimensional problem when the dispersal kernel is separable. We prove some open questions from their work on the dynamics of a single population and then extend the idea to investigate the dynamics of two spatially distributed species in (i) a competitive relation, and (ii) a predator-prey relation. In all cases, we discuss how the dynamics of the population(s) depend on the amount of suitable space that is available to them. We find a number of bifurcations, such as period-doubling sequences and Naimark-Sacker bifurcations, which we illustrate through simulations.
\end{abstract}

Keywords Integrodifference equation - Bifurcation - Separable kernel . Spatial ecology

J. Bramburger

Department of Mathematics and Statistics

University of Ottawa

Ottawa, ON, K1N8G1, Canada

Tel.: +1-613-562-5864

Fax: +1-613-562-5776

E-mail: jason_bramburger@brown.edu

Present address: Division of Applied Mathematics

Brown University, Providence, RI, 02906, USA

F. Lutscher

Department of Mathematics and Statistics and Department of Biology

University of Ottawa

Ottawa, ON, K1N8G1, Canada

Tel.: +1-613-562-5800 \# 3510

Fax: +1-613-562-5776

E-mail: flutsche@uottawa.ca 
Mathematics Subject Classification (2000) MSC 39A28 - MSC 39A60 • MSC 92B05

\section{Introduction}

Integrodifference equations are discrete-time analogues to reaction-diffusion equations [2]. Similar to their continuous-time counterparts, integrodifference equations are frequently used as models of spatial population dynamics in ecology [8]. They were developed specifically to accurately model the life cycle of organisms with separated growth and dispersal stages. The analysis of integrodifference equations, however, proves more difficult than for reactiondiffusion equations since the dynamics are discrete in time and since the equations are non-local. For that reason, simplifications, approximations, and special, explicitly solvable cases are particularly useful to have. In this paper, we formalize and extend an idea by Kot and Schaffer [8], who noticed that for a particular dispersal kernel (see below) the dynamics of the infinite-dimensional integrodifference equation reduced to a two-dimensional map. We prove an open question in their work, and we apply the method to study two systems of interacting species.

Very simply, discrete-time dynamical systems are famous for generating complex dynamics, for example the logistic map

$$
N_{t+1}=r N_{t}\left(1-N_{t}\right)
$$

that has been studied widely mathematically and used extensively in ecological modelling [12]. In this model, $N_{t}$ is the population density at time or generation $t$, and $r>0$ is the growth parameter. Alternatively, by scaling the steady-state density to unity, the logistic equation can be written as

$$
N_{t+1}=(\tilde{r}+1) N_{t}+\tilde{r} N_{t}^{2} .
$$

It is well known that the solutions to (1) converge to zero if $r<1$, and to the unique positive steady state if $1<r<3$. At $r=3$ there is the first of a cascade of period-doubling bifurcations that eventually lead to chaotic dynamics for large enough $r<4$. When $r>4$, then the equation does not preserve positivity and is therefore no longer suited to model a biological population.

Now we consider the spatial distribution of a population and denote its spatial density at time $t$ in a one-dimensional, bounded habitat $\Omega$ by $N_{t}(x)$. During the first part of their life cycle, individuals grow according to some growth function $F$ but do not move spatially. The density at the end of this growth phase is therefore $F\left(N_{t}(x)\right)$. During the second phase in the life-cycle, individuals disperse but do not reproduce or die. We denote $K(x, y)$ as the probability density of dispersal locations from initial location $y$. This function is referred to as a 'dispersal kernel' or 'seed shadow' [14]. The population density after the dispersal phase and at the beginning of the $t+1^{\text {st }}$ generation is then given by

$$
N_{t+1}(x)=\int_{\Omega} K(x, y) F\left(N_{t}(y)\right) d y .
$$


We require $K$ and $F$ to be non-negative. In general, we require $F(0)=0$, since no individuals are generated from nothing. In this paper, we will use the logistic function for $F$.

The dispersal kernel necessarily satisfies the property $\int_{\Omega} K(x, y) d x \leq 1$ for all $y \in \Omega$, since it is a probability density. Typical dispersal kernels in the ecological literature are the Gaussian and the Laplace kernels but many other forms are known and used [14]. Here, we follow the idea of Kot and Schaffer and use the cosine kernel [8]

$$
K(x, y)= \begin{cases}\frac{\pi}{4 R} \cos \left(\frac{\pi}{2 R}(x-y)\right), & |x-y|<R \\ 0, & \text { otherwise }\end{cases}
$$

The cosine dispersal kernel is separable, meaning that it can be written as

$$
K(x, y)=\frac{\pi}{4 R} \cos \left(\frac{\pi x}{2 R}\right) \cos \left(\frac{\pi y}{2 R}\right)+\frac{\pi}{4 R} \sin \left(\frac{\pi x}{2 R}\right) \sin \left(\frac{\pi y}{2 R}\right) .
$$

Throughout this work, we take the spatial domain to be an interval of length $L$, i.e. $\Omega=[-L / 2, L / 2]$. We assume that in one generation an organism can move from one point in the domain to any other. Mathematically this means that $R \geq L$. Furthermore, we can scale $R=1$ by writing $x=R \tilde{x}, y=R \tilde{y}$ and $K(x, y)=R \tilde{K}(\tilde{x}, \tilde{y})$, thus after dropping the tildes we arrive at the scaled cosine dispersal kernel:

$$
K(x, y)= \begin{cases}\frac{\pi}{4} \cos \left(\frac{\pi}{2}(x-y)\right), & |x-y|<1, \\ 0, & \text { otherwise }\end{cases}
$$

We remark that now $0 \leq L \leq 1$.

Since the cosine kernel is separable, the dynamics of the infinite-dimensional integrodifference equation can be reduced to a 2-dimensional map. We study some aspects of this map in detail in the next section. In subsequent sections, we extend the model to include two interacting species, namely competitors in section 3 , and predator and prey in section 4 .

\section{Logistic IDE}

Integro-difference equation (3) with the logistic growth function and the (scaled) separable cosine kernel was first studied by Kot and Schaeffer [8]. They noted that since the cosine kernel is separable, the dynamics of equation (3) reduce from an infinite-dimensional function space to a two-dimensional space spanned by the sine and cosine function in (5). More specifically, if we write

$$
N_{t}(x)=a_{t} \cos \left(\frac{\pi x}{2}\right)+b_{t} \sin \left(\frac{\pi x}{2}\right)
$$


then the dynamics of coefficients $a_{t}, b_{t}$ are given by the two-dimensional map

$$
\begin{aligned}
& a_{t+1}=\frac{\pi}{4} \int_{-L / 2}^{L / 2} \cos \left(\frac{\pi y}{2}\right) F\left(a_{t} \cos \left(\frac{\pi y}{2}\right)+b_{t} \sin \left(\frac{\pi y}{2}\right)\right) d y, \\
& b_{t+1}=\frac{\pi}{4} \int_{-L / 2}^{L / 2} \sin \left(\frac{\pi y}{2}\right) F\left(a_{t} \cos \left(\frac{\pi y}{2}\right)+b_{t} \sin \left(\frac{\pi y}{2}\right)\right) d y .
\end{aligned}
$$

When we choose $F$ as the logistic function (2), the two integrals become polynomials in the trigonometric functions and can be evaluated explicitly. After lengthy calculations, we arrive at the two-dimensional mapping (compare [8])

$$
\begin{aligned}
& a_{t+1}=\left(\chi_{1}-\chi_{2} a_{t}\right) a_{t}-\chi_{3} b_{t}^{2}, \\
& b_{t+1}=\left(\chi_{4}-\chi_{5} a_{t}\right) b_{t},
\end{aligned}
$$

where

$$
\begin{aligned}
& \chi_{1}=(1+r)\left[\frac{\pi L}{8}+\frac{1}{4} \sin \left(\frac{\pi L}{2}\right)\right], \\
& \chi_{2}=r \sin \left(\frac{\pi L}{4}\right)\left[1-\frac{1}{3} \sin ^{2}\left(\frac{\pi L}{4}\right)\right], \\
& \chi_{3}=\frac{1}{3} r \sin ^{3}\left(\frac{\pi L}{4}\right), \\
& \chi_{4}=(1+r)\left[\frac{\pi L}{8}-\frac{1}{4} \sin \left(\frac{\pi L}{2}\right)\right], \\
& \chi_{5}=\frac{2}{3} r \sin ^{3}\left(\frac{\pi L}{4}\right) .
\end{aligned}
$$

Kot and Schaeffer observed that the set $\left\{b_{t}=0\right\}$ is an invariant manifold of system (9), and that on this manifold, the equation for $a_{t}$ is of essentially the same form as the logistic equation in (1). It should therefore exhibit the same qualitative dynamics [8]. Furthermore, they noticed that if $-1<\chi_{4}-\chi_{5} a_{t}<1$ for all $t$, then the invariant manifold is attractive. It is clear, however, that outside of the invariant manifold, the expression for $N_{t}$ in (7) could be negative for some $x \in[-L / 2, L / 2]$ and therefore not biologically relevant.

The remainder of this section is devoted to a more thorough study of system (9). More specifically, we show that for biologically feasible parameter values $r, L$ the dynamics of (9) on the invariant manifold do, indeed, exhibit the full range of the logistic dynamics. Moreover, we show that the invariant manifold $\left\{b_{t}=0\right\}$ is locally attracting.

We begin with the fixed point $\left(a_{t}, b_{t}\right)=(0,0)$ that corresponds to the extinction of the species. Linearizing about this fixed point leads to the diagonal Jacobian matrix

$$
\left(\begin{array}{cc}
\chi_{1} & 0 \\
0 & \chi_{4}
\end{array}\right)
$$

whose eigenvalues correspond to the diagonal entries. Inspecting the expressions for $\chi_{1}$ and $\chi_{4}$ in (10), we see that $0 \leq \chi_{4} \leq \chi_{1}$ for all $r \geq 0$ and 
$L \in[0,1]$. Hence, the extinction fixed point is stable if $\chi_{1}<1$. Furthermore, since $\chi_{1}$ is an increasing function in both $r$ and $L$, there exists a unique curve in $(r, L)$-space such that $\chi_{1}(r, L)=1$. Crossing this curve leads to a loss of local stability of the extinction fixed point.

We now turn to the dynamics on the invariant manifold $\left\{b_{t}=0\right\}$. The mapping reduces to

$$
a_{t+1}=\left(\chi_{1}-\chi_{2} a_{t}\right) a_{t},
$$

which, upon applying the change of variables

$$
a_{t} \mapsto \frac{\chi_{1} a_{t}}{\chi_{2}},
$$

transforms (12) to the logistic mapping

$$
a_{t+1}=\left(\frac{\chi_{1}^{2}}{\chi_{2}}\right) a_{t}\left(1-a_{t}\right),
$$

where $\chi_{1}^{2} / \chi_{2}$ acts as the growth parameter, see (1). From the form of $\chi_{1}$ and $\chi_{2}$ given in (10) we see that for any fixed $L>0$ this growth parameter can take on any positive value as $r$ increases. Therefore, the dynamics of (12) does indeed take on the full range of logistic dynamics.

We are interested in those trajectories of (12) that remain bounded for all $t \geq 1$. We first note that any initial condition, $a_{0}$ outside of the interval $\left[0, \frac{\chi_{1}}{\chi_{2}}\right]$ maps to a negative value $a_{1}$, which in turn leads to a trajectory that remains negative for all $t \geq 1$ and diverges. Furthermore, the critical point of the mapping (12) is given by $\chi_{1}^{2} / 4 \chi_{2}$ and therefore the interval $\left[0, \frac{\chi_{1}}{\chi_{2}}\right]$ maps into itself so long as

$$
\frac{\chi_{1}^{2}}{4 \chi_{2}} \leq \frac{\chi_{1}}{\chi_{2}} \Longrightarrow \chi_{1} \leq 4 .
$$

In the complimentary case when $\chi_{1}>4$ it follows that there is an interval centred at $\chi_{1} / 2 \chi_{2}$ which maps outside of $\left[0, \frac{\chi_{1}}{\chi_{2}}\right]$. Figure 1 illustrates this behavior.

Finally, we prove that the invariant manifold is locally attracting.

Lemma 1 Let $a_{0} \in\left[0, \frac{\chi_{1}}{\chi_{2}}\right]$. If $1<\chi_{1}(r, L)<4$ then the invariant manifold $b_{t}=0$ is locally attracting.

Proof To begin, if $\chi_{1}(r, L)<4$ then those trajectories on the invariant manifold whose initial conditions, $a_{0}$, are between the roots of the logistic function satisfy

$$
0 \leq a_{t} \leq \frac{\chi_{1}^{2}}{4 \chi_{2}}
$$

for all $t \geq 1$. This implies that

$$
\chi_{4}-\chi_{5}\left(\frac{\chi_{1}^{2}}{4 \chi_{2}}\right) \leq \chi_{4}-a_{t} \chi_{5} \leq \chi_{4}
$$

for all $t \geq 1$, since the $\chi_{i}$ are positive functions of $r, L$ for $i=1, \ldots 5$. 


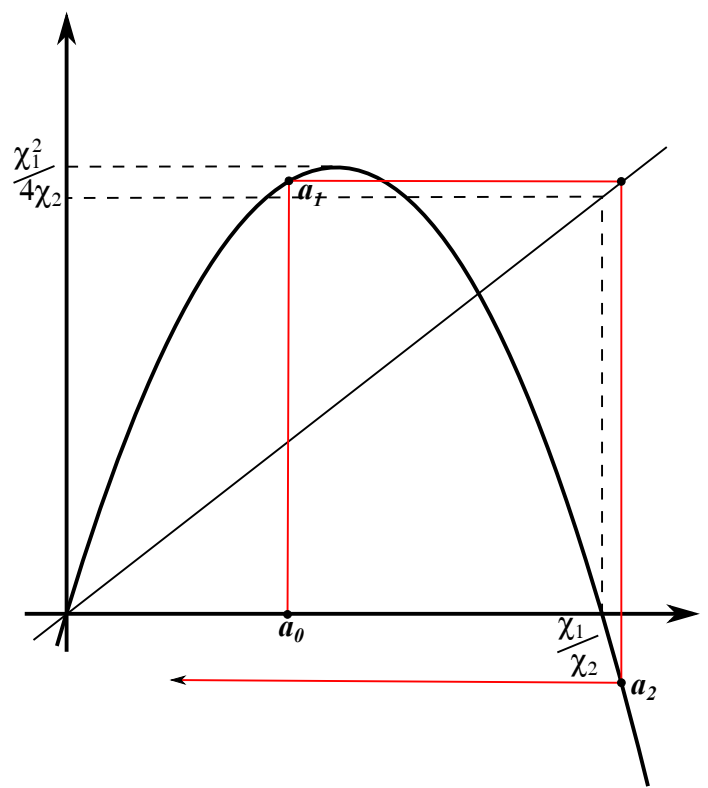

Fig. 1: Cobweb diagram of a diverging trajectory of the first component of (9) when $b_{t}=0$ and $\chi_{1}>4$.

Let us deal with the upper bound of (17) first. We wish to show that when $\chi_{1} \leq 4$ then $\chi_{4}<1$. Differentiating the function $\chi_{4}(r, L)$ with respect to $r$ and $L$ gives

$$
\begin{aligned}
& \frac{\partial \chi_{4}}{\partial r}=\left[\frac{\pi L}{8}-\frac{1}{4} \sin \left(\frac{\pi L}{2}\right)\right], \\
& \frac{\partial \chi_{4}}{\partial L}=(1+r)\left[\frac{\pi}{8}-\frac{1}{8} \cos \left(\frac{\pi L}{2}\right)\right],
\end{aligned}
$$

respectively. Both functions are strictly nonnegative for all $r \geq 0$ and $L \in[0,1]$, meaning that $\chi_{4}(r, L)$ is a nondecreasing function of both $r$ and $L$. The same can be shown for $\chi_{1}(r, L)$, meaning that for each $L, \chi_{1}(r, L)$ can only cross 4 once and $\chi_{4}(r, L)$ crosses 1 once. Therefore, if $\chi_{1}(r, L)=4$, we can solve for $r$ in terms of $L$ to get

$$
1+r^{*}(L)=\frac{4}{\frac{\pi L}{8}+\frac{1}{4} \sin \left(\frac{\pi L}{2}\right)} .
$$

Substituting this expression into $\chi_{4}(r, L)$ gives

$$
\chi_{4}\left(r^{*}(L), L\right)=\frac{4\left[\frac{\pi L}{8}-\frac{1}{4} \sin \left(\frac{\pi L}{2}\right)\right]}{\left[\frac{\pi L}{8}+\frac{1}{4} \sin \left(\frac{\pi L}{2}\right)\right]} .
$$


The function $\chi_{4}\left(r^{*}(L), L\right)$ is an increasing function of $L$, and therefore obtains its maximum on the compact interval $[0,1]$ at $L=1$. Hence,

$$
0 \leq \chi_{4}\left(r^{*}(L), L\right) \leq \chi_{4}\left(r^{*}(1), 1\right) \approx 0.88812<1 \text {. }
$$

In particular, as $r$ increases the function $\chi_{1}(r, L)$ crosses 4 before $\chi_{4}(r, L)$ crosses 1 for each $L \in[0,1]$.

Now, let us write the the lower bound of $(17)$ as $\delta(r, L)$. Then

$\delta(r, L)=(1+r)\left[\frac{\pi L}{8}-\frac{1}{4} \sin \left(\frac{\pi L}{2}\right)\right]-\frac{(1+r)^{2} \sin ^{2}\left(\frac{\pi L}{4}\right)\left[\frac{\pi L}{8}+\frac{1}{4} \sin \left(\frac{\pi L}{2}\right)\right]^{2}}{6\left[1-\frac{1}{3} \sin ^{2}\left(\frac{\pi L}{4}\right)\right]}$,

after direct substitution. This function is quadratic in $r$, and its maximum is bounded by $\chi_{4}(r, L)$ for every $L \in[0,1]$, which implies that although $\delta(r, L)$ is positive for some values of $r$ and $L$, it is always bounded above by a value less than 1 . Hence, for $L \in[0,1], \delta(r, L)$ will eventually become a strictly decreasing function for all large enough $r$. Thus, $\delta(r, L)$ can only cross -1 once.

We again substitute expression (19) to show that $\delta\left(r^{*}(L), L\right)$ is bounded below by -1 . The substitution yields

$$
\delta\left(r^{*}(L), L\right)=\frac{4\left[\frac{\pi L}{8}-\frac{1}{4} \sin \left(\frac{\pi L}{2}\right)\right]}{\left[\frac{\pi L}{8}+\frac{1}{4} \sin \left(\frac{\pi L}{2}\right)\right]}-\frac{8 \sin ^{2}\left(\frac{\pi L}{4}\right)}{3\left[1-\frac{1}{3} \sin ^{2}\left(\frac{\pi L}{4}\right)\right]},
$$

which is a decreasing function. Therefore, on the compact interval $[0,1], \delta\left(r^{*}(L), L\right)$ obtains its minimum when $L=1$. Hence,

$$
\delta\left(r^{*}(L), L\right) \geq \delta\left(r^{*}(1), 1\right) \approx-0.71188>-1 .
$$

Hence, so long as our elements $a_{t}$ remain between the roots of the scaled logistic function the invariant manifold $b_{t}=0$ is attracting. We now show that for each $a_{0} \in\left(0, \chi_{2} / \chi_{1}\right]$ we can choose $b_{0}>0$ such that $a_{t}$ will remain in the interval and therefore $b_{t}$ will converge to zero.

We write the equation for $a_{t}$ in terms of the function $f(a)=\left(\chi_{1}-\chi_{2} a\right) a$ as

$$
a_{t+1}=f\left(a_{t}\right)-\chi_{3} b_{t}^{2} .
$$

It is clear that $a_{t}$ is bounded above by $\chi_{1} / \chi_{2}$ since $\chi_{3}>0$. Clearly, given $a_{0}>0$, one can choose $b_{0}>0$ such that $a_{1}>0$. The goal is to show that $b_{t}$ approaches zero fast enough so that $a_{t}$ remains positive.

From the bounds above, we know that $b_{t}$ decays no more slowly than

$$
b_{t+1}= \pm 0.89 b_{t} .
$$


Solving this equation, we find $b_{t}^{2}=(0.89)^{2 t} b_{0}^{2}$. Substituting this expression into our equation for $a_{t+1}$ we get

$$
a_{t+1}=f\left(a_{t}\right)-\chi_{3}(0.89)^{2 t} b_{0}^{2} .
$$

The maximal value returned by $f$ is $\chi_{1}^{2} / 4 \chi_{2}$ and since $\chi_{1}<4$ we have that after one iteration of the map $f$ we have moved into a strictly smaller invariant interval, namely $\left(0, \chi_{1}^{2} / 4 \chi_{2}\right] \subset\left(0, \chi_{1} / \chi_{2}\right)$.

Since $\chi_{1}>1$ we know that the trivial equilibrium of $f$ is unstable and hence there is a region to the right of $a_{t}=0$ such that all orbits are increasing away from the origin while inside this region. Therefore, there exists an $\eta_{1}>0$ sufficiently small such that $f(a)>a$ and increasing when $a \in\left(0, \eta_{1}\right]$ and the interval $\left(\eta_{1}, \chi_{1}^{2} / 4 \chi_{2}\right]$ is mapped back into itself by $f$. Furthermore, there exists a positive $\eta_{2}<\eta_{1}$ such that $f\left(\eta_{2}\right)=\eta_{1}$. Note that $f$ maps the interval $\left(\eta_{2}, \eta_{1}\right)$ into $\left(\eta_{1}, \chi_{1}^{2} / 4 \chi_{2}\right]$. Let us write $\varepsilon_{1}:=\eta_{1}-\eta_{2}>0$ and then for any $a_{0}$ such that $f\left(a_{0}\right) \in\left(\eta_{1}, \chi_{1}^{2} / 4 \chi_{2}\right]$ we may take $b_{0}$ small enough such that

$$
0<\chi_{3} b_{0}^{2}<\varepsilon_{1} .
$$

Therefore, $a_{1}=f\left(a_{0}\right)-\chi_{3} b_{0}^{2} \in\left(\eta_{2}, \chi_{1}^{2} / 4 \chi_{2}\right]$. Moreover, since $\chi_{3}(0.89)^{2 t} b_{0}^{2} \leq$ $\chi_{3} b_{0}^{2}<\varepsilon_{1}$ we have that $a_{t+1}=f\left(a_{t}\right)-\chi_{3}(0.89)^{2 t} b_{0}^{2} \in\left(\eta_{2}, \chi_{1}^{2} / 4 \chi_{2}\right]$ for all $t \geq 1$, which remains between the roots of $f$.

We now move to those initial conditions $a_{0}$ such that $f\left(a_{0}\right) \in\left(\eta_{2}, \eta_{1}\right]$. As before, there exists a positive $\eta_{3}<\eta_{2}$ such that $f\left(\eta_{3}\right)=\eta_{2}$, and we write $\varepsilon_{2}:=\eta_{2}-\eta_{3}>0$. Then $f$ maps $\left(\eta_{3}, \eta_{2}\right]$ into $\left(\eta_{2}, \eta_{1}\right]$. We may take $b_{0}$ small enough such that

$$
0<\chi_{3} b_{0}^{2}<\varepsilon_{2}
$$

and using arguments similar to those above we must have that $a_{t+1}=f\left(a_{t}\right)-$ $\chi_{3}(0.89)^{2 t} b_{0}^{2} \in\left(\eta_{3}, \chi_{1}^{2} / 4 \chi_{2}\right]$ for all $t \geq 1$.

We may continue this process by defining $\eta_{i+1}$ strictly smaller than $\eta_{i}$ by $f\left(\eta_{i+1}\right)=\eta_{i}$ for all $i \geq 1$. Note that that $\eta_{i}>0$ at each step since $f(0)=0$ and $f(a)$ is strictly negative for $a<0$. Then for an initial condition $a_{0}$ such that $f\left(a_{0}\right) \in\left(\eta_{i+1}, \eta_{i}\right], i \geq 1$, we define $\varepsilon_{i}=\eta_{i}-\eta_{i+1}>0$ and take $b_{0}$ small enough such that

$$
0<\chi_{3} b_{0}^{2}<\varepsilon_{i} .
$$

From the previous arguments this will guarantee that $a_{t+1}=f\left(a_{t}\right)-\chi_{3}(0.89)^{2 t} b_{0}^{2} \in$ $\left(\eta_{i+1}, \chi_{1}^{2} / 4 \chi_{2}\right]$ for all $t \geq 1$. The first few iterations of this process are shown in Figure 2.

Now $\left\{\eta_{i}\right\}_{i \geq 1}$ is a monotone decreasing sequence bounded below by 0 , and is therefore convergent. If $\left\{\eta_{i}\right\}_{i \geq 1}$ converges to 0 then we have that

$$
\left(0, \chi_{1}^{2} / 4 \chi_{2}\right]=\left(\bigcup_{i=1}^{\infty}\left(\eta_{i+1}, \eta_{i}\right]\right) \cup\left(\eta_{1}, \chi_{1}^{2} / 4 \chi_{2}\right],
$$

thereby completing the proof since for each initial $a_{0}$ we can find a nontrivial interval of $b_{0}$ as above such that the invariant manifold is attracting. To see 


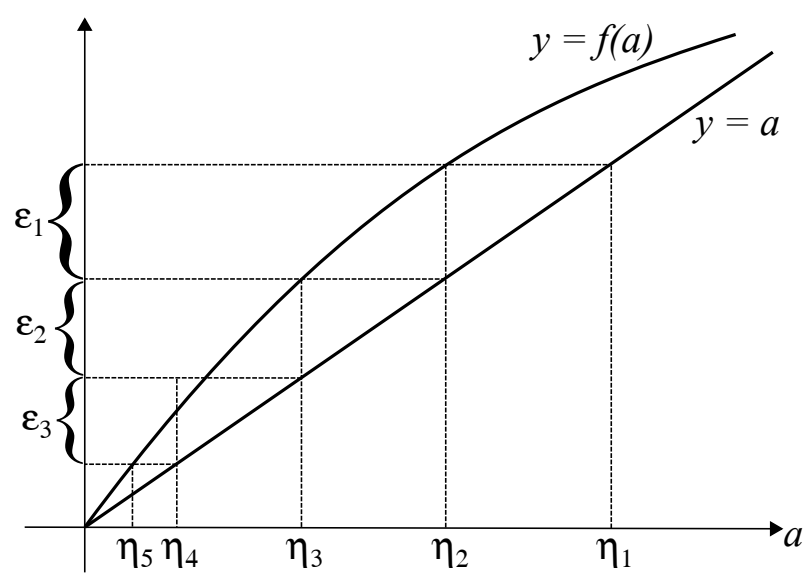

Fig. 2: The first few elements of the sequence $\left\{\eta_{i}\right\}_{i \geq 1}$.

that $\left\{\eta_{i}\right\}_{i>1}$ is converging to 0 , we assume it converges to some $\eta \neq 0$ and derive a contradiction. Immediately we can see that $\eta>0$ since the sequence must be converging to its infimum and 0 is a lower bound. But since $0<\eta<$ $\eta_{1}$ we must have that $f(\eta)>\eta$. Hence, there exists some $i \geq 1$ such that $f(\eta) \in\left(\eta_{i+1}, \eta_{i}\right]$. But this is impossible since this implies that $\eta>\eta_{i+2}$ by definition of the sequence. Hence, $\eta_{i} \rightarrow 0$ as $i \rightarrow \infty$.

Lemma 1 shows that there exists initial conditions for which solutions converge onto the invariant manifold. In particular, the full range of stable, oscillatory, and chaotic dynamics of the logistic equation can be seen in the logistic integrodifference equation as well. This behaviour was originally suggested by Kot and Schaeffer [8].

To consider ecological implications of dispersal distance and domain size, we investigate how parameters $\chi_{i}$ depend on domain size $L$. Notice that $\chi_{i}(r, 0)=0$ for each $1 \leq i \leq 5$, and all parameters are increasing in $L$. Therefore, as $L$ increases from zero, the population will at first be unable to persist until $L$ reaches a certain threshold. At this point, a globally stable, positive state will emerge. The threshold domain length is known as the 'minimal' or 'critical' domain size [15]. Increasing $L$ further will lead to instability of the positive state via period-doubling bifurcations. Eventually, the dynamics will be chaotic. Since parameters $\chi_{i}$ are also increasing in $r$, the values of $r$ at the bifurcation points decrease as $L$ increases. Put simply, the larger the domain, the smaller $r$ is required to destabilize the positive steady state and observe cyclic or chaotic solutions.

\section{Competition Model}

The ideas presented in the previous sections can be extended to models for interacting species. In particular, an integrodifference model for two species 
can be reduced to a four-dimensional model, provided both species disperse according to a cosine kernel as in (6). In this section, we consider the case of two competing species; in the next section, we will study a predator-prey system. In each case, we begin with an explanation of the non-spatial model for comparison purposes.

\subsection{Non-Spatial Model}

We extend the logistic model for a single species to two competing species, where each species reduces the per-capita growth of its competitor. Specifically, we denote by $N_{t}$ and $M_{t}$ the densities of species 1 and 2 in generation $t$. The two-dimensional mapping describing the non-spatial population dynamics is

$$
\begin{aligned}
& N_{t+1}=r N_{t}\left(1-N_{t}-\alpha M_{t}\right), \\
& M_{t+1}=s M_{t}\left(1-M_{t}-\beta N_{t}\right) .
\end{aligned}
$$

Parameters $r, s$ are the intrinsic growth parameters of Species 1 and 2, respectively. Parameters $\alpha, \beta \geq 0$ are the competition coefficients, indicating the strength with which the species affects the other. When $\alpha=\beta=0$, the equations decouple to two logistic growth models. We assume that growth parameters $r, s$ are in the interval $(0,4)$ so that the single-species models preserve positivity.

The following lemma summarizes some of the important characteristics of the mapping in (25). The proof of the lemma consists of straightforward calculations and is omitted here.

Lemma 2 Consider the mapping (25) with $\alpha, \beta \geq 0$ and $r, s \in(0,4)$.

1. If for some $t \geq 0$ we have $0 \leq N_{t} \leq 1-\alpha M_{t}$ and $0 \leq M_{t} \leq 1-\beta N_{t}$ then $0 \leq N_{t+1} \leq 1-\alpha M_{t+1}$ and $0 \leq M_{t+1} \leq 1-\beta N_{t+1}$.

2. If $r \in[0,1]$ then $N_{t} \rightarrow 0$ as $t \rightarrow \infty$.

3. If $s \in[0,1]$ then $M_{t} \rightarrow 0$ as $t \rightarrow \infty$.

The first part of the lemma identifies $\{(N, M) \mid 0 \leq N \leq 1-\alpha M, 0 \leq M \leq$ $1-\beta N\}$ as an invariant region of the mapping. This is the region in which the dynamics remain biologically relevant. When $\alpha=\beta=0$ the invariant region becomes the unit square $[0,1] \times[0,1]$. As $\alpha, \beta$ increase, the region becomes smaller.

The second and third statements of Lemma 2 give sufficient conditions for the global stability of the extinction state, $(N, M)=(0,0)$. Moreover, linearizing about the extinction state gives the sufficient condition for local stability as $r, s<1$. Therefore we see that in this case local stability implies global stability, much like in the one-dimensional logistic map.

We now turn to the three non-trivial fixed points of the model. These are the exclusion states

$$
\left(\frac{r-1}{r}, 0\right) \quad \text { and } \quad\left(0, \frac{s-1}{s}\right)
$$


along with the coexistence state

$$
\left(\frac{r \alpha(s-1)+s(1-r)}{r s(\alpha \beta-1)}, \frac{s \beta(r-1)+r(1-s)}{r s(\alpha \beta-1)}\right) .
$$

Convergence to one of the exclusion states shows that only one of the species persists in the habitat while the other goes extinct. This phenomenon is known as 'competitive exclusion'. Convergence to the coexistence state implies that both species persist in the habitat with steady population densities. We investigate the conditions for local stability of these states.

The Jacobian matrix of the mapping in (25) is given by

$$
\left[\begin{array}{cc}
r-2 r N-r \alpha M & -r \alpha N \\
-s \beta N & s-2 s M-s \beta N
\end{array}\right] .
$$

Evaluating this Jacobian matrix at the first exclusion fixed point in (26) gives

$$
\left[\begin{array}{cc}
1-r & \alpha(1-r) \\
0 & s\left(1-\beta\left(\frac{r-1}{r}\right)\right)
\end{array}\right]
$$

The eigenvalues of this upper diagonal matrix correspond to the diagonal elements, and therefore we have the following conditions for local stability of the Species 1 exclusion state:

$$
1<r<3 \quad \text { and } \quad \frac{r(s-1)}{s(r-1)}<\beta<\frac{r(s+1)}{s(r-1)} .
$$

Similarly, the stability conditions for the other exclusion state in (26) are given by

$$
1<s<3 \quad \text { and } \quad \frac{s(r-1)}{r(s-1)}<\alpha<\frac{s(r+1)}{r(s-1)} .
$$

When both of these local stability conditions are satisfied, we observe two locally stable states exist simultaneously. (Ecologists sometimes refer to this phenomenon as 'coexisting stable states' or 'bistability'.) Initial conditions will determine the outcome of competition. Alternatively, when $1<r, s<3$, $0 \leq \alpha<\frac{s(r-1)}{r(s-1)}$ and $0 \leq \beta<\frac{r(s-1)}{s(r-1)}$, neither the exclusion states nor the trivial state are stable. Local continuation theorems such as the Implicit Function Theorem allow one to see that when $(\alpha, \beta)$ are sufficiently small, a coexistence solution extends continuously from

$$
\left(\frac{r-1}{r}, \frac{s-1}{s}\right) .
$$

Since the coexistence state is stable, there is a region about $(\alpha, \beta)=(0,0)$ in which this fixed point is stable. Moreover, on the curve $\alpha=\frac{s(r-1)}{r(s-1)}$ the second exclusion state in (26) and the coexistence state meet via a transcritical bifurcation. Similarly, on the curve $\beta=\frac{r(s-1)}{s(r-1)}$ the first exclusion state in (26) 


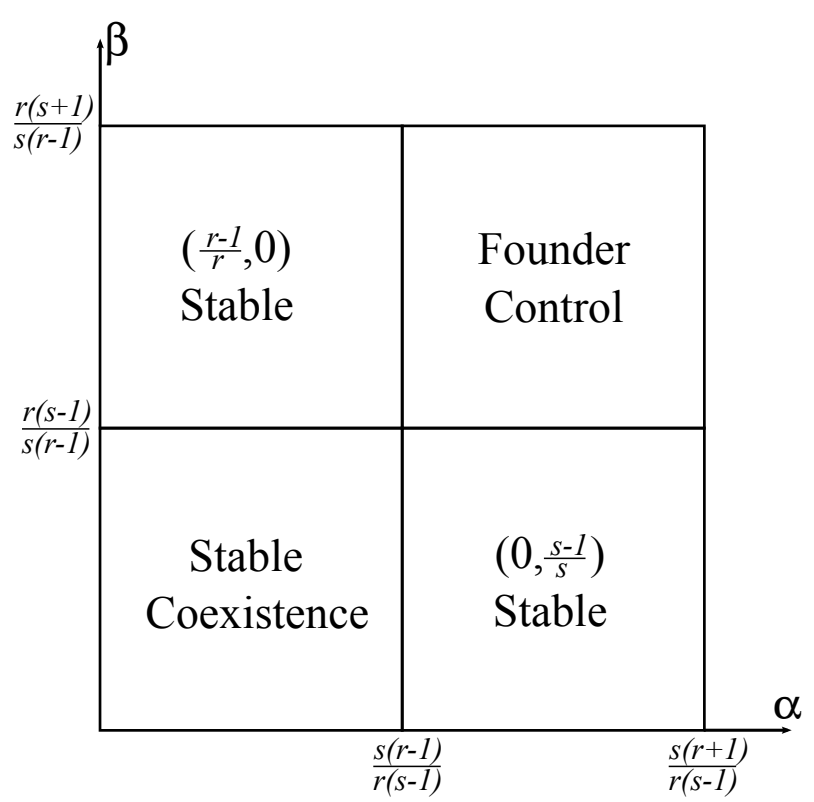

Fig. 3: Stability regions of the nontrivial fixed points of $(25)$ in $(\alpha, \beta)$ parameter space for $1<r, s<3$ fixed.

and the coexistence state meet via a transcritical bifurcation. We therefore conjecture that when neither the trivial state nor the exclusion states are stable we have a stable coexistence state. This reasoning is known in the ecological literature as the principle that 'mutual invasion implies coexistence' [4]. A stability diagram in $(\alpha, \beta)$-space is given in Figure 3 for $1<r, s<3$ fixed.

When $r, s$ exceed 3, densities begin to oscillate after undergoing a period doubling bifurcation. The analysis of these cases becomes quite complicated even in the non-spatial framework and is left for future work. Here, we limit our attention to the cases where only fixed points of the model can be stable.

The ecological interpretation of the model outcome is standard for twospecies competition models: as long as the inter-specific competition coefficients $(\alpha, \beta)$ are small enough, the two species can stably coexist. If $\beta$ is small but $\alpha$ is larger than a certain threshold, then the impact of species 2 on species 1 is so large that species 2 can exclude species 1 . Species 2 is competitively superior. If both coefficients are large, the outcome depends on initial conditions, a situation sometimes termed 'founder control'. 


\subsection{Spatial Model}

Let us now turn our attention to a system of integrodifference equations with growth function given by the competition mapping (25). That is, we consider

$$
\begin{aligned}
& N_{t+1}(x)=r \int_{\Omega} K_{N}(x, y) N_{t}(y)\left[1-N_{t}(y)-\alpha M_{t}(y)\right] d y, \\
& M_{t+1}(x)=s \int_{\Omega} K_{M}(x, y) M_{t}(y)\left[1-M_{t}(y)-\beta N_{t}(y)\right] d y,
\end{aligned}
$$

where $K_{N}$ and $K_{M}$ govern the dispersal of species 1 and 2, respectively.

We take both kernels to be cosine kernels with dispersal radii $R_{N}$ and $R_{M}$, respectively, and as before $\Omega=[-L / 2, L / 2]$, for some $L>0$. We assume that in one generation that both species can move anywhere throughout the domain, and therefore we assume without loss of generality that $R_{N} \geq R_{M} \geq L$. As before we can scale the variables appropriately to arrive at the integrodifference equation

$$
\begin{aligned}
& N_{t+1}(x)=\frac{\pi r}{4} \int_{-\tilde{L} / 2}^{\tilde{L} / 2} \cos \left(\frac{\pi}{2}(x-y)\right) N_{t}(y)\left[1-N_{t}(y)-\alpha M_{t}(y)\right] d y \\
& M_{t+1}(x)=\frac{\pi s}{4 R} \int_{-\tilde{L} / 2}^{\tilde{L} / 2} \cos \left(\frac{\pi}{2 R}(x-y)\right) M_{t}(y)\left[1-M_{t}(y)-\beta N_{t}(y)\right] d y
\end{aligned}
$$

where $R=R_{N}^{-1} R_{M}$ is the relative dispersal radius and $\tilde{L}=L R_{N}^{-1}$ the relative domain size, with $\tilde{L} \leq R \leq 1$. We will drop the tilde for ease of notation since no confusion can arise.

As before, since the cosine kernel is separable, so that we can write

$$
\begin{aligned}
& N_{t}(x)=a_{t} \cos \left(\frac{\pi x}{2}\right)+b_{t} \sin \left(\frac{\pi x}{2}\right), \\
& M_{t}(x)=c_{t} \cos \left(\frac{\pi x}{2 R}\right)+d_{t} \sin \left(\frac{\pi x}{2 R}\right) .
\end{aligned}
$$

Inserting this ansatz into integrodifference equation (34) leads to the mapping in four variables:

$$
\begin{aligned}
& a_{t+1}=\left(\xi_{1}-\xi_{2} a_{t}-\xi_{3} c_{t}\right) a_{t}-\left(\xi_{4} b_{t}+\xi_{5} d_{t}\right) b_{t}, \\
& b_{t+1}=\left(\xi_{6}-\xi_{7} a_{t}\right) b_{t}-\xi_{8} b_{t} c_{t}-\xi_{9} a_{t} d_{t} \\
& c_{t+1}=\left(\xi_{10}-\xi_{11} c_{t}-\xi_{12} a_{t}\right) c_{t}-\left(\xi_{13} b_{t}+\xi_{14} d_{t}\right) d_{t} \\
& d_{t+1}=\left(\xi_{15}-\xi_{16} c_{t}\right) d_{t}-\xi_{17} b_{t} c_{t}-\xi_{18} a_{t} d_{t},
\end{aligned}
$$


with coefficients

$$
\begin{aligned}
& \xi_{1}=r\left[\frac{\pi L}{8}+\frac{1}{4} \sin \left(\frac{\pi L}{2}\right)\right] \\
& \xi_{2}=r \sin \left(\frac{\pi L}{4}\right)\left[1-\frac{1}{3} \sin ^{2}\left(\frac{\pi L}{2}\right)\right] \\
& \xi_{3}=\frac{r R \alpha}{4}\left[2 \sin \left(\frac{\pi L}{4}\right)+\frac{1}{2 R-1} \sin \left(\frac{\pi L(2 R-1)}{4}\right)+\frac{1}{2 R+1} \sin \left(\frac{\pi L(2 R+1)}{4}\right)\right] \\
& \xi_{4}=\frac{r}{3} \sin ^{3}\left(\frac{\pi L}{4}\right) \\
& \xi_{5}=\frac{r R \alpha}{4}\left[\frac{1}{2 R-1} \sin \left(\frac{\pi L(2 R-1)}{4}\right)-\frac{1}{2 R+1} \sin \left(\frac{\pi L(2 R+1)}{4}\right)\right] \\
& \xi_{6}=r\left[\frac{\pi L}{8}-\frac{1}{4} \sin \left(\frac{\pi L}{2}\right)\right] \\
& \xi_{7}=\frac{2 r}{3} \sin ^{3}\left(\frac{\pi L}{4}\right) \\
& \xi_{8}=\frac{r R \alpha}{4}\left[-2 \sin \left(\frac{\pi L}{4}\right)+\frac{1}{2 R-1} \sin \left(\frac{\pi L(2 R-1)}{4}\right)+\frac{1}{2 R+1} \sin \left(\frac{\pi L(2 R+1)}{4}\right)\right] \\
& \xi_{9}=\xi_{5} \\
& \xi_{10}=s\left[\frac{\pi L}{8 R}+\frac{1}{4 R} \sin \left(\frac{\pi L}{2 R}\right)\right] \\
& \xi_{11}=s \sin \left(\frac{\pi L}{4 R}\right)\left[1-\frac{1}{3} \sin ^{2}\left(\frac{\pi L}{2 R}\right)\right] \\
& \xi_{12}=\frac{s \beta}{4}\left[\frac{2}{R} \sin \left(\frac{\pi L}{4 R}\right)+\frac{1}{R-2} \sin \left(\frac{\pi L(R-2)}{4 R}\right)+\frac{1}{R+2} \sin \left(\frac{\pi L(R+2)}{4 R}\right)\right] \\
& \xi_{13}=\frac{s \beta}{4}\left[\frac{1}{R-2} \sin \left(\frac{\pi L(R-2)}{4 R}\right)-\frac{1}{R+2} \sin \left(\frac{\pi L(R+2)}{4 R}\right)\right] \\
& \xi_{14}=\frac{s}{3} \sin ^{3}\left(\frac{\pi L}{4 R}\right) \\
& \xi_{15}=s\left[\frac{\pi L}{8 R}-\frac{1}{4 R} \sin \left(\frac{\pi L}{2 R}\right)\right] \\
& \xi_{16}=\frac{2 s}{3} \sin ^{3}\left(\frac{\pi L}{4 R}\right) \\
& \xi_{17}=\frac{s \beta}{4}\left[\frac{1}{R-2} \sin \left(\frac{\pi L(R-2)}{4 R}\right)-\frac{1}{R+2} \sin \left(\frac{\pi L(R+2)}{4 R}\right)\right] \\
& \xi_{18}=\frac{s \beta}{4}\left[\frac{2}{R} \sin \left(\frac{\pi L}{4 R}\right)-\frac{1}{R-2} \sin \left(\frac{\pi L(R-2)}{4 R}\right)-\frac{1}{R+2} \sin \left(\frac{\pi L(R+2)}{4 R}\right)\right]
\end{aligned}
$$

We note that $\left\{b_{t}=0, d_{t}=0\right\}$ is an invariant manifold of the mapping in (36). On this manifold, the mapping reduces to a system of competition equations almost as in (25). Since the situation is similar to that in Section 2, 
we conjecture that a proof similar to Lemma 1 can show that this manifold is locally attracting. Our work here will be restricted to this invariant manifold. Our numerical simulations indicate that it is at least locally attracting.

The mapping in (36) has several fixed points, but for the remainder of this work we will focus on only the four that relate to the four fixed points of the non-spatial model previously studied. We consider the points written as $\left(a_{t}, b_{t}, c_{t}, d_{t}\right)$ given by

$$
\begin{aligned}
& (0,0,0,0) \\
& \left(\frac{\xi_{1}-1}{\xi_{2}}, 0,0,0\right) \\
& \left(0,0, \frac{\xi_{10}-1}{\xi_{11}}, 0\right) \\
& \left(\frac{\xi_{11}\left(1-\xi_{1}\right)+\xi_{3}\left(\xi_{10}-1\right)}{\xi_{3} \xi_{12}-\xi_{2} \xi_{11}}, 0, \frac{\xi_{12}\left(\xi_{1}-1\right)+\xi_{2}\left(1-\xi_{10}\right)}{\xi_{3} \xi_{12}-\xi_{2} \xi_{11}}, 0\right) .
\end{aligned}
$$

Fixed point $(38 a)$ is the extinction state. We denote the exclusion state $(38 b)$ as $a^{*}$ and $(38 c)$ as $c^{*}$. State $(38 d)$ is the coexistence state.

Linearizing (36) about the extinction state leads to the matrix

$$
\left[\begin{array}{cccc}
\xi_{1} & 0 & 0 & 0 \\
0 & \xi_{6} & 0 & 0 \\
0 & 0 & \xi_{10} & 0 \\
0 & 0 & 0 & \xi_{15}
\end{array}\right]
$$

whose eigenvalues lie along the diagonal. So long as these diagonal elements have a modulus strictly less than 1, the trivial fixed point is stable. Moreover, from the forms given in (37) we can easily see that for all relevant parameter values

$$
0 \leq \xi_{6} \leq \xi_{1} \quad \text { and } \quad 0 \leq \xi_{15} \leq \xi_{10} .
$$

In this way, it is clear that the trivial solution is asymptotically stable if $\xi_{1}, \xi_{10}<1$.

Let us now inspect the local stability of the exclusion fixed point $a^{*}$. Linearizing about this fixed point leaves us with the upper triangular matrix

$$
\left[\begin{array}{cccc}
\xi_{1}-2 \xi_{2} a^{*} & 0 & -\xi_{3} a^{*} & 0 \\
0 & \xi_{6}-\xi_{7} a^{*} & 0 & -\xi_{9} a^{*} \\
0 & 0 & \xi_{10}-\xi_{12} a^{*} & 0 \\
0 & 0 & 0 & \xi_{15}-\xi_{18} a^{*}
\end{array}\right]
$$

where again the eigenvalues correspond to the diagonal elements. The eigenvalues coming from the first and third rows correspond to those of the non-spatial model, and therefore we focus on these exclusively since we are interested in the effect of adding a spatial aspect to our model. A necessary condition for stability of $a^{*}$ is that

$$
\left|\xi_{1}-2 \xi_{2} a^{*}\right|<1, \quad\left|\xi_{10}-\xi_{12} a^{*}\right|<1 .
$$


The first condition implies that $1<\xi_{1}<3$. In the second case, we can use the form from (37) to see that the condition is satisfied when $\beta \in\left(\beta_{\text {Lower }}, \beta_{\text {Upper }}\right)$, where

$$
\begin{aligned}
& \beta_{\text {Lower }}=\frac{\xi_{10}-1}{\frac{s}{4}\left[\frac{2}{R} \sin \left(\frac{\pi L}{4 R}\right)+\frac{1}{R-2} \sin \left(\frac{\pi L(R-2)}{4 R}\right)+\frac{1}{R+2} \sin \left(\frac{\pi L(R+2)}{4 R}\right)\right] a^{*}}, \\
& \beta_{\text {Upper }}=\frac{\xi_{10+1}}{\frac{s}{4}\left[\frac{2}{R} \sin \left(\frac{\pi L}{4 R}\right)+\frac{1}{R-2} \sin \left(\frac{\pi L(R-2)}{4 R}\right)+\frac{1}{R+2} \sin \left(\frac{\pi L(R+2)}{4 R}\right)\right] a^{*}} \text {. }
\end{aligned}
$$

Through an almost identical analysis of $c^{*}$, we find that the necessary conditions for stability which correspond to the non-spatial model are given by

$$
\left|\xi_{1}-\xi_{3} c^{*}\right|<1, \quad\left|\xi_{10}-2 \xi_{11} c^{*}\right|<1 .
$$

In this case the second condition requires that $1<\xi_{10}<3$. The first condition leads to the requirement that $\alpha \in\left(\alpha_{\text {Lower }}, \alpha_{\text {Upper }}\right)$, where

$$
\begin{aligned}
& \alpha_{\text {Lower }}=\frac{\xi_{1}-1}{\frac{r R}{4}\left[2 \sin \left(\frac{\pi L}{4}\right)+\frac{1}{2 R-1} \sin \left(\frac{\pi L(2 R-1)}{4}\right)+\frac{1}{2 R+1} \sin \left(\frac{\pi L(2 R+1)}{4}\right)\right] c^{*}}, \\
& \alpha_{\text {Upper }}=\frac{\xi_{1}+1}{\frac{r R}{4}\left[2 \sin \left(\frac{\pi L}{4}\right)+\frac{1}{2 R-1} \sin \left(\frac{\pi L(2 R-1)}{4}\right)+\frac{1}{2 R+1} \sin \left(\frac{\pi L(2 R+1)}{4}\right)\right] c^{*}} .
\end{aligned}
$$

For the remainder of this section we focus on how the stability diagram in Figure 3 changes by varying the spatial parameters $L$ and $R$. We begin by noting that if $1<\xi_{1}, \xi_{10}<3$, and both $\alpha<\alpha_{\text {Lower }}$ and $\beta<\beta_{\text {Lower }}$, then we may safely assume that the coexistence state is stable at least in the invariant $\left(a_{t}, c_{t}\right)$-plane. Furthermore, when $1<\xi_{1}, \xi_{10}<3, \alpha \in\left(\alpha_{\text {Lower }}, \alpha_{\text {Upper }}\right)$ and $\beta \in\left(\beta_{\text {Lower }}, \beta_{\text {Upper }}\right)$, then both $a^{*}$ and $c^{*}$ can be stable, leading to founder control in the model. These cases fully recover the four regions of Figure 3 in the spatial model.

Now we fix growth parameters $r, s$ and relative dispersal radius $R$ within an appropriate range and consider the stability conditions as domain length $L$ varies. Our first requirement is that $\xi_{1}, \xi_{3}>1$, as functions of just $L$. Then, since both $\xi_{1}(L)$ and $\xi_{3}(L)$ are increasing functions of $L$ on $[0, R]$, we define $L_{1}>0$ to be the first (and only) point in $[0, R]$ such that $\xi_{1}\left(L_{1}\right)=1$ and $L_{2}$ to be the first (and only) point in $[0, R]$ such that $\xi_{3}\left(L_{2}\right)=1$. Then for $L^{*}=\max \left\{L_{1}, L_{2}\right\}$ there exists a non-trivial interval in $\left(L^{*}, R\right]$ such that $1<$ $\xi_{1}, \xi_{3}<3$. Note also that functions $a^{*}(L)$ and $c^{*}(L)$ change from being negative to positive at $L_{1}$ and $L_{2}$, respectively. Therefore, $\alpha_{U p p e r}(L)$ is a decreasing function on $\left(L_{2}, R\right]$, where it is coming down from infinity at the singularity $L=L_{2}$. Similarly $\beta_{\text {Upper }}(L)$ is decreasing on $\left(L_{1}, R\right]$ where it is coming down from infinity at the singularity $L=L_{1}$. 
The lower bounds, $\alpha_{\text {Lower }}(L)$ and $\beta_{\text {Lower }}(L)$, become a bit more delicate. Just as with the upper bounds, $\alpha_{\text {Lower }}(L)$ has a singularity at $L=L_{2}$ and $\beta_{\text {Lower }}(L)$ has a singularity at $L=L_{1}$. When $L_{1}<L_{2}$ we see that $\alpha_{\text {Lower }}\left(L_{2}\right)$ is behaving in a very similar manner to $\alpha_{U \text { pper }}(L)$ on the interval $\left(L^{*}, R\right]$ since it is decreasing as we move away from the singularity at $L=L^{*}$. Now, the singularity of $\beta_{\text {Lower }}(L)$ at $L=L_{1}$ lies outside the interval of interest, but one can see that $\beta_{\text {Lower }}\left(L_{2}\right)=0$, showing that in the interval $\left(L^{*}, R\right], \beta_{\text {Lower }}(L)$ is increasing off the $L$-axis. This situation is fairly intuitive since inside the interval $\left(L_{1}, L_{2}\right)$ we have that $a^{*}(L)$ is stable and positive, whereas $c^{*}(L)$ is unstable and negative. As $L$ increases over $L_{2}$ the region in the first quadrant of $(\alpha, \beta)$-space which determined the stability of $a^{*}(L)$ continuously deforms into four separate regions to include the stability regions for a now positive and possibly stable $c^{*}(L)$. In this way, we see that by increasing the size of the spatial domain we are now able to support a wider array of dynamics and even coexistence of the species. This is summarized visually in Figure $4 a$. A similar situation holds in the case when $L_{1}>L_{2}$ and is summarized in Figure $4 b$.

Now let us inspect how the bounds on $\alpha, \beta$ change as $R$ varies. Begin by fixing $r, s$ and choose $L$ large enough so that $1<\xi_{1}<3$. Now $\xi_{10}$ is a decreasing function of $R$, and we therefore seek a point $R^{*} \geq L$ such that $1<\xi_{10}\left(R^{*}\right) \leq 3$. If such a point exists, then there exists a connected component of the interval $\left(R^{*}, 1\right]$ such that $1<\xi_{10}(R)<3$. Now as functions of $R$ over the interval $\left(R^{*}, 1\right]$ we find that both $\alpha_{\text {Upper }}(R)$ and $\alpha_{\text {Lower }}(R)$ are increasing functions. Similarly, $\beta_{\text {Upper }}(R)$ and $\beta_{\text {Lower }}(R)$ are decreasing functions of $R$ over the interval $\left(R^{*}, 1\right]$. This situation is described visually in Figure 5.

We can deduce several ecological insights from the analysis and the plots. First, we notice that $L_{1}$ and $L_{2}$ are the critical domain lengths for species $N$ and $M$, respectively. These are the minimal length of the habitat for which the species can persist in isolation (see previous section). In Figure 4a, we have $L_{1}<L_{2}$, so that species $N$ has the smaller critical domain size. While both species should benefit from a larger habitat being available to them, species $M$ benefits more in the following sense: it will require a smaller interaction strength $\left(\alpha_{\text {Lower }}\right)$ to eliminate its competitor; whereas competitor species $N$ will require a larger interaction strength $\left(\beta_{\text {Lower }}\right)$ to eliminate specie $M$. Figure $4 \mathrm{~b}$ shows the opposite behaviour.

Increasing $R$ in Figure 5 can indicate that species $M$ increases its dispersal radius or that $N$ decreases its dispersal radius. In the former case, $L_{2}$ will increase whereas in the latter $L_{1}$ will decrease. Accordingly, the results are similar to the case $L_{2}>L_{1}$ above: species $M$ benefits. The only difference to Figure $4 \mathrm{~b}$ is that the value of $\alpha_{\text {Upper }}$ now increases. This is the value where the system loses stability though a flip bifurcation. If species $M$ disperses farther, more individuals will be lost from the domain, and hence the value of $\alpha$ required to destabilize the system is higher. 


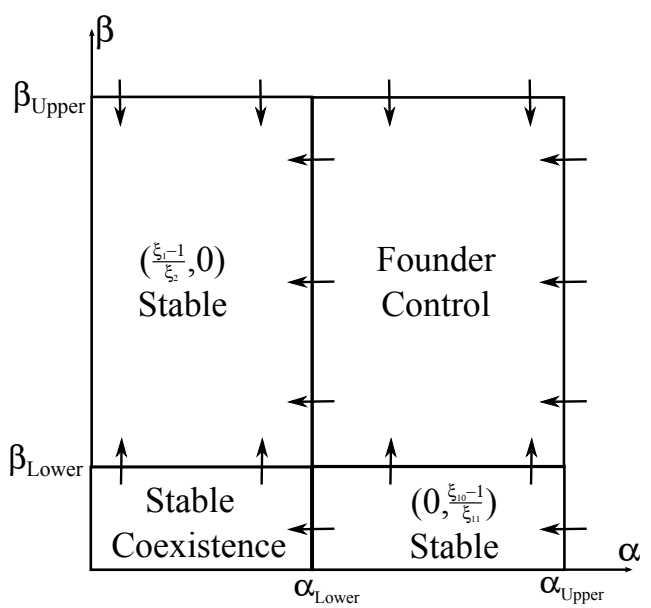

(a)

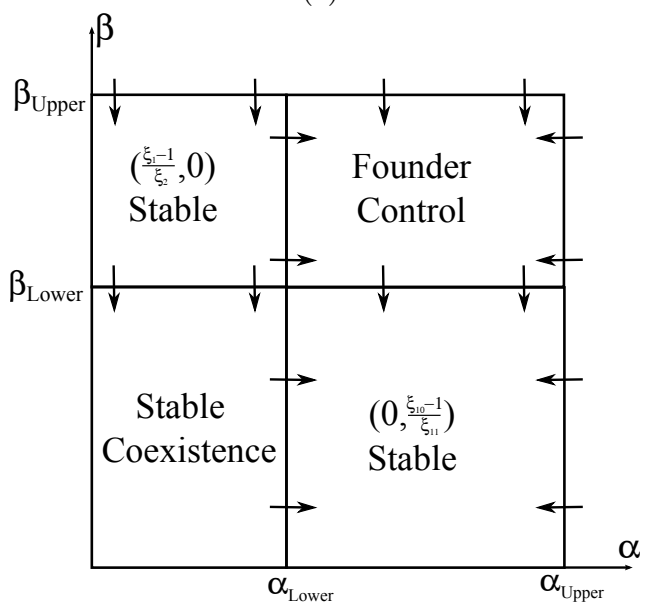

(b)

Fig. 4: Stability regions of the fixed points in $(\alpha, \beta)$ parameter space for $r, s, R$ fixed with (a) $L_{1}<L_{2}$ and (b) $L_{1}>L_{2}$. Arrows indicate the direction the bounds are moving as $L$ increases through $\left(L^{*}, R\right]$.

\section{Predator-Prey Model}

The dynamics of the competition model in the previous section are relatively simple. Only transcritical bifurcations occur in the chosen parameter range. Earlier, we had studied period doubling bifurcations in the singlesspecies model. Now we turn to a predator-prey model where we can observe a Naimark-Sacker bifurcation as well. 


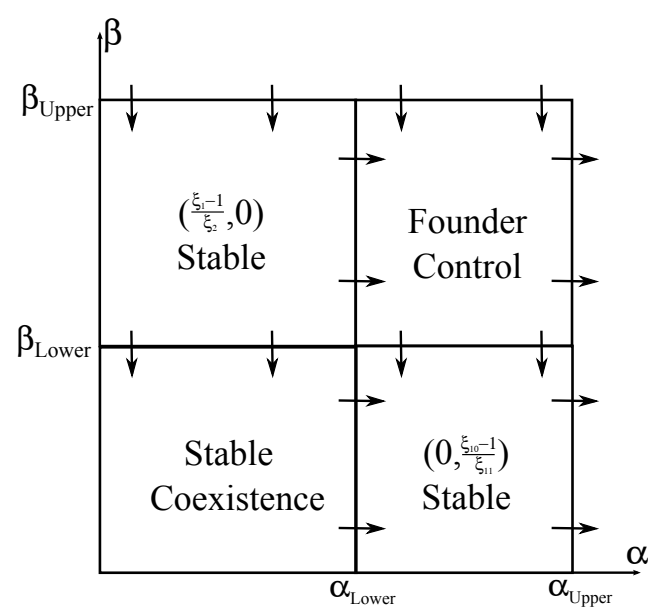

Fig. 5: Stability regions of the fixed points in $(\alpha, \beta)$ parameter space $r, s, L$ fixed and varying $R$. Arrows indicate the direction the bounds are moving as $R$ increases through $\left(R^{*}, 1\right]$.

\subsection{Non-Spatial Model}

We use a simplified (and scaled) predator-prey model, originally studied by Neubert and Kot [13], with polynomial mapping

$$
\begin{aligned}
& N_{t+1}=r N_{t}\left(1-N_{t}-P_{t}\right)_{+}, \\
& P_{t+1}=s N_{t} P_{t},
\end{aligned}
$$

where $N_{t}$ is the density of the prey and $P_{t}$ the density of the predator at generation $t$. Subscript ' + ' indicates the positive part of the expression in brackets. To avoid negative solutions, we also limit $r \in(0,4)$. Predation linearly decreases per-capita reproduction of the prey. Predator per-capita growth is linear with prey availability and growth factor $s>0$. We summarize some simple basic observations without proof in the following lemma.

Lemma 3 Consider the mapping in (46) with $r \in(0,4)$ and $s \geq 0$.

1. If $N_{t} \in[0,1]$ then $N_{t+1} \in[0,1]$ for all $t \geq 0$.

2. With initial conditions $N_{0} \in[0,1]$ and $P_{0} \geq 0$, if $s \leq 1$ then $P_{t} \rightarrow 0$ as $t \rightarrow \infty$.

3. With initial conditions $N_{0} \in[0,1]$ and $P_{0} \geq 0$, if $r \leq 1$ then $N_{t}, P_{t} \rightarrow 0$ as $t \rightarrow \infty$.

Lemma 3 gives an invariant region for the prey density and provides necessary conditions for growth. If $s<1$ then the predator approaches zero and the prey density can exhibit any of the dynamics of the logistic equation.

The mapping in (46) has three fixed points: the extinction state, $(0,0)$, the prey-only state and the coexistence state. A detailed bifurcation analysis of 
this non-spatial model (in a slightly different parametrization) was given in [13]. We summarize the most important results for later reference.

The prey-only state is given explicitly by

$$
\left(\frac{r-1}{r}, 0\right)
$$

and the coexistence state by

$$
\left(\frac{1}{s}, \frac{s(r-1)-r}{s r}\right) .
$$

The Jacobian matrix of system (46) has the form

$$
\left(\begin{array}{cc}
r(1-2 N-P) & -r N \\
s P & s N
\end{array}\right)
$$

The extinction state is locally stable when $r<1$, and, by Lemma 3 , it is also globally stable in this case. The stability region for the prey-only state is given by

$$
s<\frac{r}{r-1}, \quad 1<r<3 .
$$

Crossing the curve $r=1$ leads to a transcritical bifurcation from extinction to prey persistence. Crossing the curve $r=3$ with $s<r /(r-1)$ leads to a perioddoubling bifurcation from the prey persistence state. Numerical simulations reveal that this bifurcation leads to temporal oscillations in just the prey while the predator remains absent.

The Jacobi matrix at the coexistence state (48) is given by

$$
\left(\begin{array}{cc}
r\left(1-\frac{2}{s}-\frac{s(r-1)-r}{s r}\right. & \frac{-r}{s} \\
\frac{s(r-1)-r}{r} & 1
\end{array}\right) .
$$

The Jury conditions [6] lead to the following stability region (see also [13])

$$
\frac{r}{r-1}<s<\frac{2 r}{r-1}, \quad s>\frac{3 r}{r+3}, \quad 1<r<4 .
$$

Crossing the curve $s=r /(r-1)$, for $1<r<3$, leads to a transcritical bifurcation in which the prey persistence state loses stability to the coexistence state. Moreover, crossing the curve $s=2 r /(r-1), 1<r \leq 4$, leads to a Neimark-Sacker bifurcation in which the coexistence state loses stability to a closed invariant curve. We observe temporally oscillating population densities of both species. Crossing the curve $s=3 r /(r+3)$ for $3<r<4$ leads to a period doubling bifurcation in which also the predator species vanishes from the system [13]. This behavior was termed the 'subcritical collapse of predator populations'. The stability results are summarized in Figure 6. 


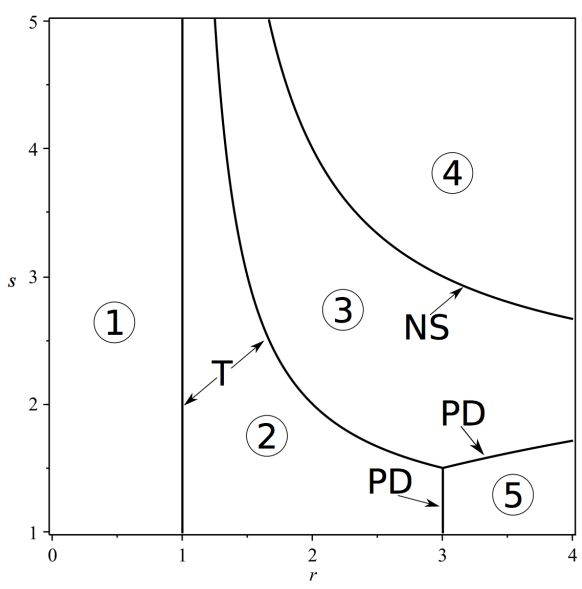

Fig. 6: Bifurcation diagram in parameter space for the mapping (46). Bifurcation curves are marked as: T - transcritical, PD - period doubling, NS Neimark-Sacker. In Region 1 the extinction state is stable. In Region 2 prey persistence is stable. Coexistence of the species is stable in Region 3. Region 4 has a stable invariant curve in $\left(N_{t}, P_{t}\right)$-space. In Region 5 there is a succession of period doubling bifurcations from the prey persistence state leading to chaos.

\subsection{Spatial Model}

Having explored the bifurcation structure of the non-spatial model, we turn to the corresponding integrodifference model to study how spatial dispersal and domain size affect predator-prey dynamics. After scaling (see previous section), we consider the system

$$
\begin{aligned}
& N_{t+1}(x)=\frac{\pi r}{4 R} \int_{\frac{-L}{2}}^{\frac{L}{2}} \cos \left(\frac{\pi}{2 R}(x-y)\right) N_{t}(y)\left(1-N_{t}(y)-P_{t}(y)\right) d y, \\
& P_{t+1}(x)=\frac{\pi s}{4} \int_{\frac{-L}{2}}^{\frac{L}{2}} \cos \left(\frac{\pi}{2}(x-y)\right) N_{t}(y) P_{t}(y) d y,
\end{aligned}
$$

where $R=R_{N} R_{P}^{-1}$ is the relative dispersal radius of prey to predator and $L \leq R \leq 1$ is the scaled domain size. We assumed that the predator disperses further than the prey.

As before, we simplify the infinite-dimensional system to a finite-dimensional map by using separability of the cosine kernel. Inserting a similar ansatz to 
the one in (35) into (53) leads to the mapping in four variables:

$$
\begin{aligned}
a_{t+1} & =\left(\zeta_{1}-\zeta_{2} a_{t}-\zeta_{3} c_{t}\right) a_{t}-\zeta_{4} b_{t}^{2}-\zeta_{5} b_{t} d_{t}, \\
b_{t+1} & =\left(\zeta_{6}-\zeta_{7} a_{t}\right) b_{t}-\zeta_{8} b_{t} c_{t}-\zeta_{9} a_{t} d_{t}, \\
c_{t+1} & =\zeta_{10} a_{t} c_{t}+\zeta_{11} b_{t} d_{t}, \\
d_{t+1} & =\zeta_{12} b_{t} c_{t}+\zeta_{13} a_{t} d_{t},
\end{aligned}
$$

with

$$
\begin{aligned}
& \zeta_{1}=r\left[\frac{\pi L}{8 R}+\frac{1}{4} \sin \left(\frac{\pi L}{2 R}\right)\right] \\
& \zeta_{2}=r \sin \left(\frac{\pi L}{4 R}\right)\left[1-\frac{1}{3} \sin ^{2}\left(\frac{\pi L}{4 R}\right)\right] \\
& \zeta_{3}=\frac{r}{4}\left[\frac{1}{R} \sin \left(\frac{\pi L}{4 R}\right)+\frac{1}{R-2} \sin \left(\frac{\pi L(R-2)}{4 R}\right)+\frac{1}{R+2} \sin \left(\frac{\pi L(R+2)}{4 R}\right)\right] \\
& \zeta_{4}=\frac{r}{3} \sin ^{3}\left(\frac{\pi L}{4 R}\right) \\
& \zeta_{5}=\frac{r}{4}\left[\frac{1}{R-2} \sin \left(\frac{\pi L(R-2)}{4 R}\right)-\frac{1}{R+2} \sin \left(\frac{\pi L(R+2)}{4 R}\right)\right] \\
& \zeta_{6}=r\left[\frac{\pi L}{8 R}-\frac{1}{4} \sin \left(\frac{\pi L}{2 R}\right)\right] \\
& \zeta_{7}=\frac{2}{3} r \sin ^{3}\left(\frac{\pi L}{4 R}\right) \\
& \zeta_{8}=\frac{r}{2}\left[\frac{2}{R} \sin \left(\frac{\pi L}{4 R}\right)-\frac{1}{R-2} \sin \left(\frac{\pi L(R-2)}{4 R}\right)-\frac{1}{R+2} \sin \left(\frac{\pi L(R+2)}{4 R}\right)\right] \\
& \zeta_{9}=\frac{r}{4}\left[\frac{1}{R-2} \sin \left(\frac{\pi L(R-2)}{4 R}\right)-\frac{1}{R+2} \sin \left(\frac{\pi L(R+2)}{4 R}\right)\right] \\
& \zeta_{10}=\frac{s R}{4}\left[2 \sin \left(\frac{\pi L}{4 R}\right)+\frac{1}{2 R-1} \sin \left(\frac{\pi L(2 R-1)}{4 R}\right)+\frac{1}{2 R+1} \sin \left(\frac{\pi L(2 R+1)}{4 R}\right)\right] \\
& \zeta_{11}=\frac{s R}{4}\left[\frac{1}{2 R-1} \sin \left(\frac{\pi L(2 R-1)}{4 R}\right)-\frac{1}{2 R+1} \sin \left(\frac{\pi L(2 R+1)}{4 R}\right)\right] \\
& \zeta_{12}=\frac{s R}{4}\left[\frac{1}{2 R-1} \sin \left(\frac{\pi L(2 R-1)}{4 R}\right)-\frac{1}{2 R+1} \sin \left(\frac{\pi L(2 R+1)}{4 R}\right)\right] \\
& \zeta_{13}=\frac{s R}{4}\left[2 \sin \left(\frac{\pi L}{4 R}\right)-\frac{1}{2 R-1} \sin \left(\frac{\pi L(2 R-1)}{4 R}\right)-\frac{1}{2 R+1} \sin \left(\frac{\pi L(2 R+1)}{4 R}\right)\right]
\end{aligned}
$$

As before, we find that $b_{t}=d_{t}=0$ is an invariant manifold of the mapping and we will see again that the behaviour on this invariant manifold closely resembles that of the non-spatial model. The relevant fixed points are the extinction state, the prey-only state, and the coexistence state, which we can 
find explicitly (written as $\left.\left(a_{t}, b_{t}, c_{t}, d_{t}\right)\right)$ :

$$
\begin{aligned}
& (0,0,0,0) \\
& \left(\frac{\zeta_{1}-1}{\zeta_{2}}, 0,0,0\right) \\
& \left(\frac{1}{\zeta_{10}}, 0, \frac{\zeta_{10}\left(\zeta_{1}-1\right)-\zeta_{2}}{\zeta_{3} \zeta_{10}}, 0\right)
\end{aligned}
$$

The trivial fixed point is stable when $\zeta_{1}<1$. As in the non-spatial model, if prey growth rate $r$ is chosen small enough, then the extinction state is stable, independently of domain size and dispersal radius.

Linearizing about the prey-only state, (56b), gives the matrix

$$
\left(\begin{array}{cccc}
2-\zeta_{1} & 0 & \frac{\zeta_{3}\left(1-\zeta_{1}\right)}{\zeta_{2}} & 0 \\
0 & \zeta_{6}-\frac{\zeta_{7}\left(\zeta_{1}-1\right)}{\zeta_{2}} & 0 & \frac{\zeta_{9}\left(1-\zeta_{1}\right)}{\zeta_{2}} \\
0 & 0 & \frac{\zeta_{10}\left(\zeta_{1}-1\right)}{\zeta_{2}} & 0 \\
0 & 0 & 0 & \frac{\zeta_{13}\left(\zeta_{1}-1\right)}{\zeta_{2}}
\end{array}\right)
$$

which is an upper-diagonal matrix, meaning that its eigenvalues are the diagonal elements. The eigenvalues coming from the first and third row correspond to those of the non-spatial model, and therefore we focus on these eigenvalues exclusively. Hence, a necessary condition for the stability of the fixed point $(56 \mathrm{~b})$ is

$$
\left|2-\zeta_{1}\right|<1 \quad \text { and } \quad\left|\frac{\zeta_{10}\left(\zeta_{1}-1\right)}{\zeta_{2}}\right|<1 .
$$

The first condition implies that $1<\zeta_{1}<3$. To understand the second expression, we use the explicit formulae in (55). Since $\zeta_{10}$ depends linearly on the growth parameter $s$, the latter condition is satisfied when $0<s<s_{1}$, where

$$
s_{1}=\frac{4 \zeta_{2}}{R\left(\zeta_{1}-1\right)\left[2 \sin \left(\frac{\pi L}{4 R}\right)+\frac{1}{2 R-1} \sin \left(\frac{\pi L(2 R-1)}{4 R}\right)+\frac{1}{2 R+1} \sin \left(\frac{\pi L(2 R+1)}{4 R}\right)\right]},
$$

which is implicitly a function of $r$.

Holding $L$ and $R$ fixed, we are able to plot this region in parameter space for $s$ as a function of $r$. To begin we set $r_{1}$ to be the unique positive value of $r$ such that $\zeta_{1}=1$. That is,

$$
r_{1}=\frac{1}{\left[\frac{\pi L}{8 R}+\frac{1}{4} \sin \left(\frac{\pi L}{2 R}\right)\right]} .
$$

Similarly, we set $r_{2}$ to be the unique point such that $\zeta_{1}=3$, which leads to the bounds $r_{1}<r<r_{2}$. One can easily see from the form of $r_{1}$ (and by extension $r_{2}$ ) that as $L$ increases $r_{1}$ (and $r_{2}$ ) decrease. In the same way, if $R$ increases then $r_{1}$ (and $r_{2}$ ) increase as well. One sees that $s_{1}$ has a singularity at 
$r=r_{1}$, where $s_{1}$ is decreasing to the right of $r_{1}$ for the duration of the interval $\left(r_{1}, r_{2}\right)$. Numerical investigations indicate that holding $R$ fixed and increasing $L$ through the interval $(0, R]$ the curve $s_{1}$ is decreasing, and thus the region becomes smaller for larger $L$. Similar investigations reveal that when $L$ is fixed and $R$ varies through the interval $[L, 1]$ the curve $s_{1}$ is increasing, and thus the region becomes larger for larger $R$.

The linearization of the coexistence point (56c) is given by the matrix

$$
\left(\begin{array}{cccc}
\frac{\zeta_{10}-\zeta_{2}}{\zeta_{10}} & 0 & \frac{-\zeta_{3}}{\zeta_{10}} & 0 \\
0 & \zeta_{6}-\frac{\zeta_{7}}{\zeta_{10}}-\frac{\zeta_{8} \zeta_{10}\left(\zeta_{1}-1\right)-\zeta_{2} \zeta_{8}}{\zeta_{3} \zeta_{10}} & 0 & \frac{-\zeta_{9}}{\zeta_{10}} \\
\frac{\zeta_{10}\left(\zeta_{1}-1\right)-\zeta_{2}}{\zeta_{3}} & 0 & 1 & 0 \\
0 & \frac{\zeta_{12}\left[\zeta_{10}\left(\zeta_{1}-1\right)-\zeta_{2}\right]}{\zeta_{10} \zeta_{3}} & 0 & \frac{\zeta_{13}}{\zeta_{10}}
\end{array}\right) .
$$

The eigenvalues of this matrix are quite complicated and for this reason we restrict our analysis to the invariant manifold $b_{t}=d_{t}=0$. This reduces the $4 \times 4$ matrix to the $2 \times 2$ matrix

$$
\left(\begin{array}{cc}
\frac{\zeta_{10}-\zeta_{2}}{\zeta_{10}} & \frac{-\zeta_{3}}{\zeta_{10}} \\
\frac{\zeta_{10}\left(\zeta_{1}-1\right)-\zeta_{2}}{\zeta_{3}} & 1
\end{array}\right)
$$

allowing us to now use the Jury conditions to inspect the stability. In this case the Jury conditions lead to

$$
\begin{aligned}
& \zeta_{1}-\frac{2 \zeta_{2}}{\zeta_{10}}<1, \\
& \zeta_{1}-\frac{\zeta_{2}}{\zeta_{10}}>1, \\
& \zeta_{1}-\frac{3 \zeta_{2}}{\zeta_{10}}>-3 .
\end{aligned}
$$

Simplifying the first two conditions and using our previous notation of the curve $s_{1}$ and the values $r_{1}$ and $r_{2}$ gives $s_{1}<s<2 s_{1}$ on the interval $r_{1}<r \leq r_{2}$. On the interval $r \geq r_{2}$ the third Jury condition becomes important and the lower bound on $s$ now becomes

$$
s_{2}=\frac{12 \zeta_{2}}{R\left(\zeta_{1}+3\right)\left[2 \sin \left(\frac{\pi L}{4 R}\right)+\frac{1}{2 R-1} \sin \left(\frac{\pi L(2 R-1)}{4 R}\right)+\frac{1}{2 R+1} \sin \left(\frac{\pi L(2 R+1)}{4 R}\right)\right]},
$$

greatly resembling the condition for stability of the coexistence state in the non-spatial model. Moreover, this curve behaves in a similar way to that of $s_{1}$ in that holding $R$ fixed and varying $L$ through the interval $(0, R]$ causes the curve to decrease and holding $L$ fixed and varying $R$ through the interval $[L, 1]$ cause the curve to increase.

Therefore we see that on the invariant manifold $b_{t}=d_{t}=0$ the behaviour of the spatial model greatly mimics that of the non-spatial model. A bifurcation 


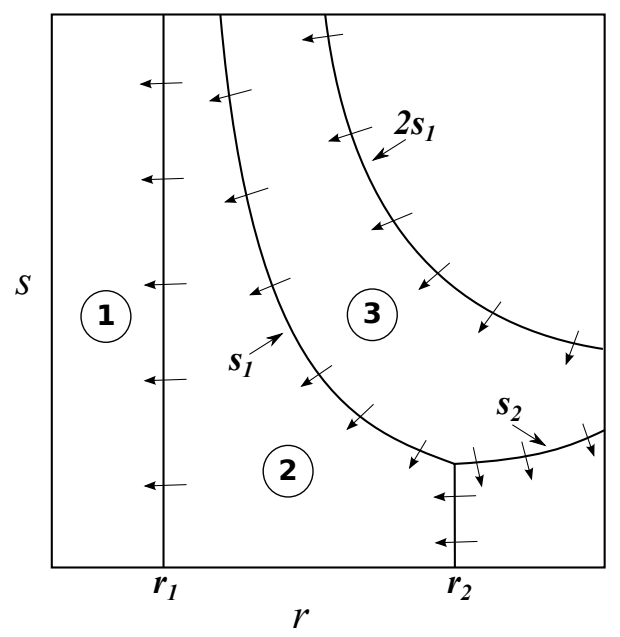

Fig. 7: Bifurcation diagram in parameter space for the mapping (54) on the invariant manifold $b_{t}=d_{t}=0$. In Region 1 the extinction state is stable. Prey persistence is stable in Region 2 and coexistence is stable in Region 3. The arrows on the bifurcation curve represent the direction the curves are moving when $R$ is fixed and $L$ increases through the interval $(0, R]$. When $L$ is fixed and $R$ increases through the interval $[L, 1]$ all arrows reverse directions.

diagram on this invariant manifold looks roughly the same as that of the nonspatial model given in Figure 6 and has corresponding steady-states stable in the various regions. In this way we observe that the introduction of space does not qualitatively change the dynamics of the model, at least on an invariant manifold. The stability results presented here are summarized in Figure 7 along with the effect the additional spatial variables, $R$ and $L$, have on the bifurcation curves.

The arrows in Figure 7 indicate that increasing the domain size in a spatial predator-prey model has roughly the same effect as increasing the prey growth rate in the non-spatial model: when $L$ is larger then a smaller value of $r$ is required for prey persistence, for predator persistence and for the existence of stable oscillations. Vice versa, increasing the relative dispersal radius has the opposite effect of increasing the domain size. These relationships show that the dynamics of this predator-prey system are determined by the 'effective' prey growth rate, in some sense the portion of prey growth that remains within the domain. Increasing the domain size or decreasing prey dispersal radius increases this effective growth rate of the prey since fewer individuals leave the domain due to dispersal.

In Figure $8 \mathrm{~b}$ we provide numerically generated orbit diagrams of the full mapping (54) to demonstrate that stability on the invariant manifold seems to imply stability within the full mapping (54). For the chosen values $L=$ 0.85, $R=0.9$, we have $r_{1} \approx 1.613$ and $r_{2} \approx 4.839$. In Figure 8a we hold 


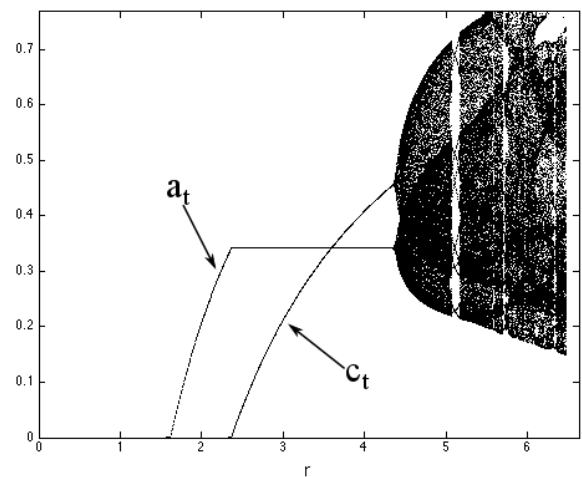

(a)

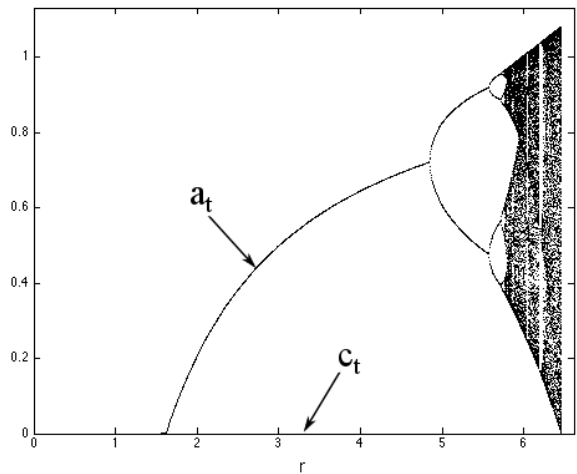

(b)

Fig. 8: Simulations of the full mapping (54) with $r$ increasing up from 0 . Parameters are set to $L=0.85, R=0.9$ and (a) $s=5.5$, (b) $s=2$. The limiting values of $a_{t}$ and $c_{t}$ are plotted for each value of $r$.

$s=5.5$ and plot the limiting values of $a_{t}$ and $c_{t}$ using the initial conditions $\left(a_{0}, b_{0}, c_{0}, d_{0}\right)=(0.1,0.1,0.1,0.1)$ for each $r$. One sees that the trivial solution attracts the initial conditions for $0<r<r_{1}$ and as $r$ crosses $r_{1}$ a transcritical bifurcation occurs leading to the attraction by the prey persistence state. Solving $s_{1}(r)=5.5$ with $L=0.85$ and $R=0.9$ gives $r \approx 2.358$, which from Figure $8 \mathrm{a}$ we can see is the place when the coexistence state begins attracting initial conditions. From the Jury conditions, we find that crossing $s=2 s_{1}$ leads to a Neimark-Sacker bifurcation which gives temporally oscillating prey and predator densities. Solving $2 s_{1}(r)=5.5$ for $r$ with $L=0.85$ and $R=0.9$ gives $r \approx 4.384$, which we again see is reflected in Figure $8 \mathrm{a}$.

In Figure $8 b$ we use the same technique as that used to generate Figure $8 a$, but taking $s=2$. As expected, we see the prey-only state bifurcate at $r=r_{1}$. However, we have chosen $s$ so small that the coexistence state does 
not emerge. From the stability conditions of the prey persistence state on the invariant manifold we saw that crossing $r=r_{2}$ with $s<s_{2}\left(r_{2}\right)$ leads to a eigenvalue of -1 which gives a period-doubling bifurcation. This perioddoubling bifurcation can be observed in Figure 8b, where we see that further increasing $r$ leads to a period-doubling cascade into chaos with the component $a_{t}$ mimicking the dynamics of the logistic function.

\section{Discussion}

Integrodifference equations model the density of populations with discrete, non-overlapping generations in a continuous spatial habitat. This modelling framework is very appealing to ecologists since many species, in particular in temperate climates, show distinct growth and dispersal phases during their life cycle. In addition, and in contrast to reaction-diffusion equations [2], the framework easily accommodates very general descriptions of dispersal behaviour through the use of different dispersal kernels [14]. Even empirically obtained kernels without given parametric representation can be directly substituted into the equations.

At the same time, the mathematical theory of the dynamical behaviour of integrodifference equations is still relatively young - it began with the work by Kot and Schaeffer [8] — and incomplete. Discrete dynamical systems are known to exhibit more complex behaviour than their continuous-time analogues, as is obvious when comparing the discrete logistic equation (1) with any one-dimensional continuous equation. The integral operator in (3) makes the analysis only harder. Over the years, researchers have developed various techniques to analyze stability and other properties of integrodifference equations.

Kot and Schaeffer suggested the use of a separable kernel to reduce the infinite-dimensional integrodifference equation to a 2-dimensional map [8]. They conjectured, but did not prove, that in certain cases, the dynamics of the map approach a 1-dimensional manifold, on which the dynamics of the logistic map are recovered. We proved that this manifold is, indeed, locally attracting for the map. We also showed that by varying the original model parameters (scaled $r$ and $L$ ), the dynamics on the invariant manifold show the complete range of the logistic map. We found that increasing growth parameter $r$ has the same qualitative effect as increasing scaled domain size $L$ (and therefore also the same as decreasing non-scaled dispersal radius $R$ ). Hence, the dynamics are determined by some 'effective growth parameter' that measures the growth in population that remains in the domain after dispersal, rather than being lost from the domain via movement. While our technique is specific to the separable kernel, the overall results are not. They have been shown for different kernels, for example the Laplace kernel, where the eigenvalue problem can be reduced to a boundary-value problem $[8,16]$. The idea of an 'effective growth parameter' also arises in an approximation technique for integrodifference equations, known as 'average dispersal success', and leads to the same 
qualitative results $[5,11,17]$. Other authors have used the idea of reduction of dimensionality and approximated kernels via separable kernels [9].

To our knowledge, nobody has used the approach of dimensionality reduction to study the effects of spatial domain size and dispersal range on interacting species. In fact, relatively few papers deal with integrodifference equations for interacting populations on bounded domains $[1,3,7,8,10,14]$. Our results on the effect of domain size on predator-prey dynamics are analogous to those found by Cobbold et al. who used the dispersal success approximation for the Laplace kernel [3]. Some aspects of how dispersal behaviour affects the outcome of competition were studied via the average dispersal success in [10].

There are, of course, limits to the method of dimension reduction. Mathematically, the simple spatial shape of solutions in the ansatz (35) implies that on the invariant manifold, the spatial distribution of the population is always a cosine function, in particular always concave. It is known that this is not the case for a simple integrodifference equation with logistic growth function and Gaussian or Laplace dispersal kernel [11]. Similarly, several aspects of interacting populations, such as pattern formation in predator-prey systems [14], cannot be observed with the cosine kernel. From a modelling point of view, the cosine kernel is clearly only a special case that does not approximate all natural dispersal behaviour well. Based on our results here, we suggest that using a low-order approximation, such as simply doubling the ansatz in (35) with different radii, to a Laplace or Gaussian kernel could lead to an analytically tractable model that could capture the most important aspects. We leave this idea for future work as well as the extension of the proof that the invariant manifold is locally attracting also in the higher-dimensional cases.

\section{References}

1. Allen, E., Allen, L., Gilliam, X.: Dispersal and competition models for plants. J. Math. Biol. 34, 455-481 (1996)

2. Cantrell, R.S., Cosner, C.: Spatial Ecology via Reaction-Diffusion Equations. Mathematical and Computational Biology. Wiley (2003)

3. Cobbold, C., Lewis, M., Lutscher, F., J., R.: How parasitism affects critical patch size in a host-parasitoid system: application to Forest Tent Caterpillar. Theor. Pop. Biol. 67(2), 109-125 (2005)

4. Durrett, R.: Mutual Invadability Implies Coexistence in Spatial Models. American Mathematical Society (2002)

5. Fagan, W., Lutscher, F.: The average dispersal success approximation: A bridge linking home range size, natal dispersal, and metapopulation dynamics to critical patch size and reserve design. Ecol. Appl. 16(2), 820-828 (2006)

6. Jury, E.: Inners and Stability of Dynamic systems. Wiley (1974)

7. Kot, M.: Diffusion-driven period-doubling bifurcations. BioSystems 22, 279-287 (1989)

8. Kot, M., Schaffer, W.M.: Discrete-time growth-dispersal models. Math. Biosc. 80, 109136 (1986)

9. Latore, J., Gould, P., Mortimer, A.: Spatial dynamics and critical patch size of annual plant populations. J. theor. Biol. 190, 277-285 (1998)

10. Lutscher, F.: Density-dependent dispersal in integrodifference equations. J. Math. Biol. 56(4), 499-524 (2008)

11. Lutscher, F., Lewis, M.: Spatially-explicit matrix models. A mathematical analysis of stage-structured integrodifference equations. J. Math. Biol. 48, 293-324 (2004) 
12. May, M.: Stability and Complexity in Model Ecosystems. Princeton Univ. Press (1973)

13. Neubert, M., Kot, M.: The subcritical collapse of predator populations in discrete time predator-prey models. Mathematical Biosciences 110, 45-66 (1992)

14. Neubert, M., Kot, M., Lewis, M.A.: Dispersal and pattern formation in a discrete-time predator-prey model. Theor. Pop. Biol. 48(1), 7-43 (1995)

15. Skellam, J.G.: Random dispersal in theoretical populations. Biometrika 38, 196-218 (1951)

16. Van Kirk, R.W.: Integrodifference models for biological growth and dispersal. Ph.D. thesis, University of Utah (1995)

17. Van Kirk, R.W., Lewis, M.A.: Integrodifference models for persistence in fragmented habitats. Bull. Math. Biol. 59(1), 107-137 (1997) 